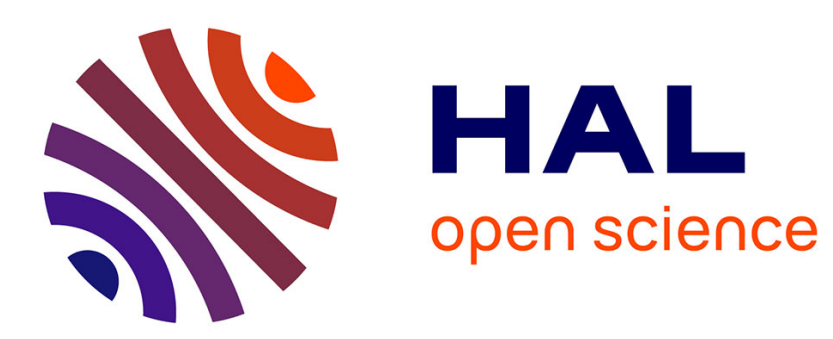

\title{
Organicisme et théorie des classes sociales chez Simiand et Halbwachs : un héritage caché de Durkheim ? \\ Jean-Christophe Marcel
}

\section{To cite this version:}

Jean-Christophe Marcel. Organicisme et théorie des classes sociales chez Simiand et Halbwachs : un héritage caché de Durkheim ?. Revue d'histoire des sciences humaines, 2008, 19 (2), pp.143 - 143. 10.3917/rhsh.019.0143 . hal-01625748

\section{HAL Id: hal-01625748 \\ https://hal.science/hal-01625748}

Submitted on 28 Oct 2017

HAL is a multi-disciplinary open access archive for the deposit and dissemination of scientific research documents, whether they are published or not. The documents may come from teaching and research institutions in France or abroad, or from public or private research centers.
L'archive ouverte pluridisciplinaire HAL, est destinée au dépôt et à la diffusion de documents scientifiques de niveau recherche, publiés ou non, émanant des établissements d'enseignement et de recherche français ou étrangers, des laboratoires publics ou privés. 


\section{ORGANICISME ET THÉORIE DES CLASSES SOCIALES CHEZ SIMIAND ET HALBWACHS : UN HÉRITAGE CACHÉ DE DURKHEIM ? \\ Jean-Christophe Marcel}

Ed. Sc. Humaines | « Revue d'Histoire des Sciences Humaines »

2008/2 n 19 | pages 143 à 160

ISSN 1622-468X

ISBN 2912601834

Article disponible en ligne à l'adresse :

http://www.cairn.info/revue-histoire-des-sciences-humaines-2008-2-page-143.htm

\section{!Pour citer cet article :}

Jean-Christophe Marcel, «Organicisme et théorie des classes sociales chez Simiand et Halbwachs : un héritage caché de Durkheim? », Revue d'Histoire des Sciences Humaines 2008/2 (n 19), p. 143-160.

DOI 10.3917/rhsh.019.0143

Distribution électronique Cairn.info pour Ed. Sc. Humaines.

(C) Ed. Sc. Humaines. Tous droits réservés pour tous pays.

La reproduction ou représentation de cet article, notamment par photocopie, n'est autorisée que dans les limites des conditions générales d'utilisation du site ou, le cas échéant, des conditions générales de la licence souscrite par votre établissement. Toute autre reproduction ou représentation, en tout ou partie, sous quelque forme et de quelque manière que ce soit, est interdite sauf accord préalable et écrit de l'éditeur, en dehors des cas prévus par la législation en vigueur en France. Il est précisé que son stockage dans une base de données est également interdit. 


\title{
Organicisme et théorie des classes sociales chez Simiand et Halbwachs : un héritage caché de Durkheim ?
}

\author{
Jean-Christophe MARCEL
}

\begin{abstract}
Résumé
En reprenant les références faites par Durkheim à l'organicisme social dans La division $d u$ travail social, cet article interroge les bases épistémologiques de la théorie des classes sociales élaborée plus tard par François Simiand et Maurice Halbwachs. Il en ressort que dans la continuité de la morphologie sociale durkheimienne la société de classes de Simiand et Halbwachs peut aussi être comprise comme une organisation aux prises avec la lutte pour l'existence, s'efforçant de s'adapter à son environnement. Dans cette lutte toutes les classes ne sont pas à égalité, certaines jouant un rôle plus essentiel au maintien de l'intégrité de la société. Il en résulte que la hiérarchie sociale peut aussi être pensée en termes d'évolution différenciée de types sociaux plus ou moins engagés dans le mouvement de la civilisation.
\end{abstract}

Mots-clés : Durkheim - Halbwachs - Simiand - Classe sociale - Organisation Évolution.

\begin{abstract}
Organicism and Social claSses Theory. From Durkheim to Simiand and Halbwachs : a Hidden Legacy?

Considering the use Durkheim made of the organicist theory in his book about The Division of Labour, this article aims at questioning the epistemological grounds of Halbwachs' and Simiand's theories of social classes. One could read them as a continuity of Durkheim's former program of social morphology, where society is an organisation struggling for life. In this struggle, social classes are several social types, more or less important to impulse the vital movement of the society. In this way civilization is the evolution of the society trying to acclimate its groups to the natural and social environment.
\end{abstract}

Key-words : Durkheim - Halbwachs - Simiand - Social Classes - Organization Evolution. 


\section{Introduction}

Dans un article récent, D. Guillo met en évidence, à la suite de C. Blanckaert, la place de la biologie dans les premiers écrits de Durkheim ${ }^{1}$. Se dégage ainsi une vision durkheimienne de la société conçue comme une « organisation, c'est-à-dire une forme matérielle apte à accomplir un certain nombre de fonctions vitales ${ }^{2}{ }^{2}$. Il en résulterait une théorie des différents types de sociétés se distinguant par un degré variable de différenciation, lui-même adossé à un critère morphologique : la plus ou moins grande densité morale et matérielle de la société. Ainsi, le passage de la fameuse société à solidarité mécanique au groupe à solidarité organique se décline comme la modification de la forme de la société sous l'effet de la division du travail et de la différenciation ${ }^{3}$. Ce thème, répandu dans la France du XIX ${ }^{\mathrm{e}}$ siècle renvoie à la question de l'adaptation des organismes sous la contrainte de la lutte vitale. La société est alors comparable à un organisme qui entretient des relations obligées à ses « conditions d'existence », tant internes qu'externes, pour reprendre un thème cher à Cuvier ${ }^{4}$.

On en déduit alors une échelle des types d'organisation des sociétés, conçue comme une « échelle de développement». Ainsi, Durkheim rapproche-t-il « l'échelle sociale » de « l'échelle animale » afin d'établir une typologie des " espèces sociales » conforme à l'évolution ${ }^{5}$. Le développement des sociétés depuis les sociétés segmentaires jusqu'aux formes les plus développées de la civilisation se calque si l'on peut dire sur le mode «agglomérant des colonies animales » (51). Plus le type social est développé, plus la différenciation est grande, plus la civilisation s'affranchit du corps, ou si l'on préfère de l'organisme en tant qu'il est chose naturelle inséré dans, et soumis à, la matière et devient une chose sociale ${ }^{6}$. Il en résulte qu'au niveau individuel, la vie instinctive s'affaiblit au et à mesure qu'on s'élève dans l'échelle des civilisations, et donc des types humains qui en résultent : à mesure que les sociétés se différencient le développement psychique des individus s'affranchit des penchants héréditaires instinctuels pour se constituer en s'appuyant sur des potentialités multiples, indéfinies, plus complexes et plus délicates, c'est-à-dire susceptibles de s'adapter aux multiples aléas de l'existence (525).

" À mesure que l'on s'élève vers les types humains « supérieurs », se développe et s'étend une sphère de la conscience de plus en plus propre à l'individu, individualisée, sous l'effet, en grande partie du milieu professionnel » (527).

En somme, individus (et sociétés), en évoluant - en se différenciant pour échapper à la concurrence -, s'émanciperaient progressivement des contraintes structurelles imposées par tout ce qui est morphologique, c'est-à-dire ce qui renvoie au corps des hommes - et à celui de la société, i.e. la façon dont elle s'étend et se répartit dans l'espace. Alors que dans les sociétés segmentaires à solidarité mécanique, les « sauvages » mus par une forte conscience collective se ressemblent tous et sont au regard de la division du travail dans une relative situation d'interchangeabilité, dans

\footnotetext{
${ }^{1}$ Principalement De la division du travail social et Les règles de la méthode sociologique.

${ }^{2}$ BlancKaert, 2004, Chapitre III ; GuILlo, 2006, 516.

${ }^{3}$ GuILlo, 2006, 516.

${ }^{4}$ Pour une présentation des grandes lignes de la théorie de Cuvier, $c f$. BLANCKAERT, 2004, 36-37.

${ }^{5}$ BLANCKAERT, 2004, 50-51.

${ }^{6}$ Guillo, 2006, 525.
} 
les sociétés modernes le processus d'individuation issu de la différenciation fonctionnelle entraîne un développement différenciée des individualités parallèle à ce que l'on pourrait nommer le développement de la socialité.

Nous souhaiterions, dans ce texte, donner quelques pistes pour montrer que ce paradigme peut éclairer la théorie des classes sociales dans la sociologie économique de Simiand et Halbwachs.

Il semble en effet que dans la lignée de Durkheim l'un et l'autre conçoivent la marche vers la civilisation comme une suite d'actions que la société opère sur son substrat (sa population et l'inscription de cette dernière dans l'espace) pour s'adapter aux transformations du milieu dans lequel elle évolue. Dans cette lutte pour l'existence, les classes sociales, et la façon dont elles entrent en contact les unes avec les autres, jouent un rôle crucial. Cependant, l'avènement de la civilisation urbaine semble ne pas profiter à toutes, et c'est comme si on pouvait voir en les classes sociales différents types d'organisation, plus ou moins évoluées selon leur plus ou moins grande dépendance à l'égard de la nature, ou ce qui revient au même, leur plus ou moins grande proximité avec ce qui fait à un moment donné l'essence de la vie sociale. De la sorte on pourrait s'autoriser à lire la théorie dite du «foyer » chez Halbwachs, et la théorie de l'action économique chez Simiand comme un prolongement de la théorie morphologique chez Durkheim, et du paradigme organiciste qu'on y trouve.

\section{I - Civilisation, marche du progrès et lutte pour la vie}

\section{Genre de vie, suicide et civilisation}

Dans Les causes du suicide (1930), Halbwachs se livre à un réexamen de statistiques du suicide pensé comme un symptôme permettant d'appréhender la façon dont la société vit le passage d'un mode de civilisation à un autre. Ce dernier peut avantageusement être caractérisé par le concept de genre de vie, défini par Halbwachs comme "un ensemble de coutumes, de croyances et de manières d'être, qui résultent des occupations habituelles des hommes et de leur mode d'établissement», si bien que «deux genres de vie ou deux types de civilisation, quelque différence qu'il y ait entre eux, se ressemblent en ce qu'ils comportent un nombre plus ou moins grand d'occasions pour les hommes d'entrer en rapport les uns avec les autres, rapports amicaux, rapports indifférents ou rapports d'hostilité »" ${ }^{7}$.

Or, là où c'est le genre de vie urbain qui prévaut, on se suicide beaucoup plus. Et de manière générale les différents taux tendent à s'harmoniser en se calquant sur celui que dicte la vie urbaine. Le suicide est donc un bon indicateur du passage d'une civilisation à une autre, car le genre de vie urbain s'oppose au genre de vie rural, comme la vie moderne s'oppose à la vie d'antan, en somme un type social à un autre. À la campagne en effet, la vie collective est à la fois très forte et très simplifiée. Les occupations et les événements sont plus restreints, car, dans ce monde, vie professionnelle et vie familiale sont peu dissociées. Les oppositions creusent des abîmes plus forts, si bien qu'il existe plus de spontanéité, d'élan primitif et de sauvagerie. Mais en revanche les occasions de heurts sont moins nombreuses. Dans la

\footnotetext{
${ }^{7}$ Halbwachs, 1930, 502.
} 
société urbaine, « non seulement les lieux où se déroule l'activité professionnelle sont distincts et d'ordinaire éloignés dans l'espace des maisons qui constituent le cadre matériel de la vie domestique, mais encore les périodes consacrées à ces deux modes d'existence se trouvent nettement séparées et n'empiètent pas l'une sur l'autre » (505506). Dans ces deux sphères de la vie, un courant plus rapide passe entre les hommes, une plus grande diversité de situations plus ou moins durables se concentrent dans un même temps. En ville la vie sociale se déroule à un rythme plus intense, en même temps que le morcellement dans l'espace provoque en elle un morcellement équivalent : bref, moins de sauvagerie, des représentations collectives plus floues et plus complexes.

\section{Lutte pour la vie, complication sociale}

Les motifs de suicide, bien sûr, naissent comme tous les autres de ces types de rapports qui, en se multipliant et s'intensifiant, influencent les pensées et les sentiments des hommes ${ }^{8}$.

Dans la ville, si la vie collective est plus intense, c'est parce qu'elle est prise dans un réseau de voies où la circulation est elle-même plus intense que nulle par ailleurs. Il en résulte un mélange de représentations collectives qui font que les groupements sociaux ont tendance à s'y dissoudre plus qu'ailleurs. Dans le monde économique, on voit que les diverses professions prennent conscience d'elles-mêmes dès lors qu'elles sont rangées dans certaines parties de l'espace telles les rues commerçantes ou les rues de métiers. Mais, en même temps, la proximité avec d'autres plus haut placées dans l'échelle sociale met sous les yeux des individus l'image hiérarchisée des corps et leur rappelle leur relative indigence.

Ainsi, les occasions sont donc renforcées pour chacun d'éprouver un sentiment collectif plus puissant qu'ailleurs, lié à des masses d'hommes dont on ne perçoit pas les limites, mais aussi, parallèlement, les probabilités sont plus grandes de sentir un sentiment d'isolement extrême, comme on le verra dans le cas des ouvriers. Les revers de fortune, ennuis et déceptions de carrière «se produisent plus fréquemment dans une société plus complexe, où les situations individuelles changent plus souvent et plus vite, où le rythme de la vie est plus rapide, où il y a plus de risques pour les individus de se trouver désadaptés par rapport à leur milieu ${ }^{9}{ }^{9}$. Dans les sociétés modernes, plus individualistes, les individus sont livrés à eux-mêmes et donc plus vulnérables au suicide, parce que la vie devient plus compliquée. La hausse des suicides est le produit de tendances stables, la conséquence de cette plus ou moins grande complexification, de la substitution d'un genre de vie urbain à un genre de vie rural, laquelle est à comprendre comme le processus par lequel un mode de régulation sociale succède à un autre. Elle est la contrepartie d'une « complication nécessaire qui est la condition d'une vie sociale plus riche et plus intense ${ }^{10}$. En d'autres termes, les tendances collectives au suicide et leur évolution sont révélatrices de l'arrivée

\footnotetext{
${ }^{8}$ Pour plus de renseignements sur la théorie chez Halbwachs des états psychiques collectivement constitués, $c f$. MARCEL, 2004.

${ }^{9}$ HaLBWACHS, 1939, 13.

${ }^{10}$ Ibid., 13-14.
} 
de types sociaux plus complexes nés de processus de régulation nécessaires à la gestion de cette complexité.

\section{Individualisme}

Cette discipline se caractérise principalement par le développement d'une mentalité qui attache plus de prix à l'existence individuelle et à sa prolongation. En d'autres termes, la société, tout entière tournée vers sa propre conservation, ne peut perdurer dans un type de civilisation urbain que si elle exerce sur son « corps »- les individus mis au contact les uns des autres en d'autres termes la population -, une pression qu'il faut interpréter comme le processus par lequel elle s'efforce de s'adapter aux conditions de son milieu, selon un processus qu'on peut assimiler à une sorte de compétition vitale. Ce sont les individus les plus compétitifs qui survivent, les autres sont impitoyablement éliminés : "les vaincus de la vie forment ainsi une longue cohorte de captifs que la société traîne derrière son char ${ }^{11}$.

Ainsi s'explique aussi le progrès de la limitation des naissances, qui est une réaction instinctive face au manque de place qui caractérise la nouvelle structure de population qu'est la ville. Quand beaucoup d'enfants naissent, tous ne peuvent pas entrer tout de suite en ménage. Dans une population composée de peu d'enfants au contraire, la formation d'un ménage se heurte à moins d'obstacles parce qu'elle représente un changement moins important. La famille conjugale est un mode d'union issus d'aptitudes que la société moderne s'efforce d'extirper de certains hommes afin d'adapter les conditions de la vie familiale au milieu. La baisse de la mortalité est aussi à considérer comme le résultat d'une volonté de durer et de se préoccuper de la valeur de l'existence individuelle, que la société impulse à ses membres.

«Autrefois, la société n'hésitait pas à gaspiller ses membres, parce qu'elle ne trouvait point d'obstacle à les remplacer (...). Le renouvellement des générations était alors bien plus rapide. Aujourd'hui, la société s'intéresse surtout à elle-même, mais, pour cette raison même, elle se désintéresse de moins en moins des individus, ou, du moins, elle à intérêt à ce que les individus se préoccupent de plus en plus d'eux-mêmes, à ce que la valeur de l'existence individuelle soit de plus en plus appréciée ${ }^{12}$.

La lutte organisée contre la mort résulte de ce que dans la ville, la société fait en sorte que les hommes prennent de plus en plus conscience de leur individualité. Nous sommes dans la droite ligne de ce qu'énonce Durkheim. Les individus ont besoin de sauvegarder la liberté de leurs mouvements et leur faculté de participer en personne à toutes les activités, ce qui les conduit à alléger leurs charges et leurs entraves. Et, comme une vie sociale plus compliquée n'est supportable que par ceux qui ne sont pas trop longtemps et trop gravement accablés par les déconvenues que leur réserve l'existence, la société tolère d'autant moins la présence de certains qui développent des sentiments nuisibles à son fonctionnement. En conséquence elle ne fait rien pour les retenir à la vie : elle essaie d'exercer son influence sur le nombre de ses morts en préservant ceux qui l'aident à conserver son intégrité, et

\footnotetext{
${ }^{11}$ HALBWACHS, 1930, 461.

${ }^{12}$ Halbwachs, 1970a, 128-129.
} 


\section{en éliminant impitoyablement les autres. Le groupe est mû par un instinct de conservation si fort qu'il peut pousser à tuer.}

«Toutes les fonctions de la société s'orientent vers la vie et vers elle seule. Le groupe ne meurt pas ; ce sont ses membres, les individus qui, de temps en temps, s'éliminent, et, pourvu qu'ils soient remplacés, il n'y a pas à s'en soucier. On peut appliquer à la société ce qu'on a toujours pensé des dieux, savoir qu'ils diffèrent principalement des hommes en ce que la mort ne les atteint pas » (121).

\section{Les données immédiates de la conscience sociale}

Le fait d'habiter à la ville ou à la campagne, d'exercer tel ou tel métier ne provoque pas chez les individus la même conscience d'appartenance au groupe, de par les modifications de la disposition dans l'espace des activités sociales. En ce sens la forme matérielle du groupe est à la source de la vie psychologique "première » du collectif des individus qui le composent : ce sont les images spatiales qui vont donner naissance aux états psychologiques collectivement constitués, plus ou moins compliqués, qui motivent les individus, parce qu'elles sont à l'origine des représentations les plus essentielles que le groupe se fait de lui-même.

«Comprenons bien (...) que les formes matérielles de la société agissent sur elle, non point en vertu d'une contrainte physique, comme un corps agirait sur un autre corps, mais par la conscience que nous en prenons, en tant que membres d'un groupe qui perçoivent son volume, sa structure physique, ses mouvements dans l'espace. Il y a là un genre de pensée ou de perception collective, qu'on pourrait appeler une donnée immédiate de la conscience sociale, qui tranche sur toutes les autres » (182-183) ${ }^{13}$.

La société, mue par une sorte "d'instinct de conservation », sait ce qui est bon pour elle et insuffle en toutes circonstances à ses membres un élan vital qui travaille à sa perpétuité. Il existe en effet,

«Une routine possible, une imagination, ou une illusion commune au groupe (...) un sens intuitif et profond, un instinct collectif qui équivaut à une sagesse supérieure, sens des conditions d'un équilibre, sens du caractère presque indéfini de la prise que la population, mais elle seule (...) peut avoir sur elle-même » (176).

En bref, pour qu'il puisse se faire une idée de ce qui est nécessaire à sa perpétuation, un groupe doit commencer par se faire la représentation la plus claire possible de lui-même. D'où la relation privilégiée qu'il entretient avec les formes matérielles dans lesquelles il s'incarne : leur relative fixité lui fournit, en plus d'une preuve tangible de son existence, un principe originel de stabilité. En effet, ses membres peuvent alors se le représenter plus clairement et ressentir plus fortement leur communauté d'appartenance. Une fois construites, ces formes spatiales ont leur dynamisme propre, mais elles évoluent très progressivement, si bien qu'alors que les hommes passent et meurent, la société ne s'éteint pas avec eux. Elle dure. Elle conserve une autonomie, une existence propre qui lui permet de continuer à s'imposer aux esprits.

«En d'autres termes, de même qu'un corps vivant est soumis en partie aux conditions de la matière inerte, parce que, par tout un aspect de lui-même, il est une chose matérielle, une société, réalité psychique, ensemble de pensées et tendances collectives (...) a cependant un corps organique, et participe aussi à la nature des choses

${ }^{13}$ Chacun aura relevé ici l'allusion à Bergson. 
physiques. C'est pourquoi elle s'enferme, à certains égards, elle se fixe dans des formes, dans des arrangements matériels qu'elle impose aux groupes dont elle est faite» (168).

Dans toute activité qu'elle développe, la pensée du groupe prend conscience de son corps et adapte son organisation aux possibilités qu'elle perçoit, « tout comme la pensée individuelle a besoin de percevoir le corps et l'espace pour se maintenir en équilibre $\gg{ }^{14}$. Mais cette prise de conscience s'effectue de manière différente selon les fonctions qu'occupent les groupes dans la société.

\section{Les classes sociales : un type social prépondérant dans une organisation évoluée}

Dans le prolongement de ces vues sur l'organisation et son évolution, la prééminence des classes sociales dans les sociétés modernes peut être pensée comme le produit de la structure la plus évoluée en terme de différenciation des fonctions. Ainsi, chez Simiand ce sont le genre de vie et la répartition des dépenses « un certain ensemble d'habitudes pour l'emploi même de ces revenus, notamment la nature et les catégories de dépenses, la proportion des diverses catégories de dépenses $"{ }^{15}$ qui sont l'indicateur d'une conscience de classe qui sert de fondement collectif à des aspirations à tel ou tel genre de vie, des résistances à tel ou tel changement. Par ailleurs, dans chaque société, c'est dans le plan qui paraît le plus important du point de vue de l'organisation sociale que se définissent les classes sociales. Ici se trouve le critère distinctif des taxinomies :

«En effet, de façon générale, les classes se constituent dans le plan de ce qui dans une société est le plus apprécié, de ce qui donne la considération, de ce qui comporte la puissance, l'autorité, les moyens de vie, d'action, soit réels, soit estimés tels » (442).

Dès lors, c'est sur le plan économique que l'on doit apprécier le rôle et la place des classes dans la hiérarchie dans une société d'échange complexe.

« Ce nous paraît être un grand fait très manifeste et très peu contestable que le fait économique proprement dit, pourvu d'une existence bien distincte et de caractère bien différencié, est, comme tel, un fait relativement récent dans les sociétés humaines, à la différence d'autres catégories de phénomènes sociaux (...). En aucune autre société que dans nos sociétés occidentales modernes, et surtout contemporaines, le fait économique n'est apparu plus nettement et plus distinctement constitué, en même temps que plus important » (582-583).

En d'autres termes les classes sociales sont les types sociaux issus de la division du travail la plus poussée qui soit. Dans le cadre de la sociologie économique des sociétés complexes, la définition de ce type passe selon Simiand par une mentalité économique particulière, dont l'importance se mesure au rôle qu'elle tient dans l'impulsion qu'elle donne au mouvement économique, lequel est réductible à la démarche que suit la société pour s'adapter à son milieu et réguler la lutte pour la vie. En somme, comme chez Claude Bernard, la compétition vitale est médiatisée par un ensemble de régulations nées des interactions particulières qu'exige la vie économique.

\footnotetext{
${ }^{14}$ Ibid., 12-13.

${ }^{15}$ SimiAND, 1930, 446.
} 


\section{Raison collective et cycle économique}

Or, ce mouvement se caractérise par l'alternance entre des phases de récession et d'expansion qui constituent une série de cycles économiques, laquelle alternance est le résultat de la confrontation entre l'action des travailleurs et celle des entrepreneurs. De cette confrontation, naît l'accroissement des richesses et des revenus, qui est un fait nouveau propre aux types sociaux caractéristiques des sociétés les plus développées ${ }^{16}$. Il en résulte une accélération du développement économique qui est le résultat de la réaction mutuelle des entrepreneurs et des ouvriers, suite à une variation de la quantité de monnaie en circulation.

Ce résultat n'est pas voulu individuellement, ni recherché par les hommes dans leur activité de tous les jours, précise Simiand. Ces mouvements et comportements sociaux sont entraînés

«Par une sorte d'instinct de vie, favorable en somme au développement du groupe, même sans pleine conscience chez les individus ou (...) plus exactement, par une raison collective meilleure et plus efficacement appliquée aux réalités majeures ${ }^{17}$.

Les variations de prix et salaires qui résultent de ces mouvements mettent en jeu la perception qu'ont les groupes en présence de leur place dans l'espace social. Nous avions déjà constaté ce parallèle avec les vues d'Halbwachs : le milieu social imprime dans les consciences des besoins et aspirations qui travaillent à la conservation de la société ${ }^{18}$. Or, bien que ce soit moins clairement exprimé que chez Halbwachs, on trouve aussi dans le modèle de Simiand la conception d'une hiérarchie sociale dont le critère est la plus ou moins grande proximité des classes concernées avec les représentations collectives que cela met en jeu: ici principalement celles que véhiculent la monnaie comme on va le voir. Dans ce dernier cas, c'est la proximité plus ou moins grande avec les arcanes du monde économique qui fonde la hiérarchie sociale. Mais cette proximité est une affaire de plus ou moins grande compatibilité « instinctuelle » pourrait-on dire.

\section{II - Classes sociales, adaptation au milieu et lutte pour la vie}

\section{Les aspects de l'instinct social}

La théorie des classes sociales d'Halbwachs développe cette thématique, quoique sous un autre jour. L'influence du milieu social se fait sentir à partir du moment où le groupe imprime sa marque sur la nature des individus, en la modelant en fonction de ses fins, qui sont réductibles à la démarche qu'il suit pour s'adapter à son milieu. Plus cette démarche est élaborée, plus le groupe s'émancipe de sa dépendance à l'égard de sa forme matérielle, plus ses membres étouffent leurs instincts naturels au profit de leurs instincts sociaux. On retrouve exactement les résultats énoncés par Durkheim dans la Division du travail.

"Quelque compte qu'il faille tenir des dispositions naturelles des individus, et des différences entre eux, au sein d'un même groupe, qui ne tiennent en effet qu'à eux et à

\footnotetext{
${ }^{16}$ MARCEL, 2001.

${ }^{17}$ SIMIAND, 1932b, 2, 518.

${ }^{18}$ Marcel, Steiner, 2006, 18.
} 
leur organisme, reconnaissons en tous cas qu'elles n'auraient pu s'épanouir, et ressortir avec un tel relief, sans un autre "climat», c'est-à-dire si l'individu ne s'était point trouvé pris en tel milieu ou telle organisation sociale $»{ }^{19}$.

L'instinct de survie, plus ou moins « socialisé » en quelque sorte, s'exprime au sein des classes sociales en motifs, aspirations, dont la forme phénoménale prend l'aspect de l'esprit de famille, de l'ambition, de l'épargne, de la recherche de biens matériels etc. "leur forme, leur intensité », "résultent des conditions propres au groupe, de sa structure et de ses rapports avec les autres » (58). Ces motifs constituent comme l'indicateur du stade de développement propre à un type de classe sociale donnée.

«Les hommes (...) se présenteront à nous dans leurs formes de vie naturelles et propres à nous révéler, en la plénitude de ses instincts et de ses tendances, la nature humaine qui est une nature sociale ${ }^{20}$.

Ils expriment en même temps la façon dont la classe sociale insuffle à ses membres la « sagesse supérieure » qui, dans la lutte pour la survie, travaille au mieux à sa perpétuation. Les différents stades d'évolution de ces groupes sociaux dépendent explicitement du rapport qu'ils entretiennent avec la matière chez Halbwachs, comme on sait. Plus le métier rattache l'homme aux objets inertes, et moins il a la possibilité d'accéder à la « socialité ».

Dans la lutte pour la vie que suppose la marche vers le progrès tous ainsi ne sont pas à égalité. Et, de ce point de vue, la différenciation durable entre fonctions de direction et d'exécution paraît centrale pour comprendre la stratification sociale dans les sociétés d'échange complexe : on conditionne ou on est plus ou moins conditionné par les mouvements économiques, qui sont autant d'adaptation que la société réalise pour durer dans le temps et dans l'espace. Si dans la théorie de Simiand le rapport à la matière semble évacué, on le retrouve toutefois au travers d'une certaine capacité à agir sur un environnement le plus social qui soit : le monde économique, car c'est le plus changeant, mais aussi le plus chargé en représentations collectives complexes et variées. Plus on peut agir dessus en interprétant les signaux de prix qu'il renferme, et plus on a accès à la «socialité ». En d'autres termes, plus sa «mentalité économique ${ }^{21}$ permet d'interpréter efficacement valeurs et représentations économiques et d'agir efficacement sur elles, plus la classe considérée a une place de choix dans la compétition vitale.

\section{III - En bas de la hiérarchie sociale : des groupes moins « évolués »?}

\section{Les paysans la terre, et la nature}

Dans la lignée de la différence explicitée plus haut entre civilisations urbaine et rurale, les classes paysannes portent la marque du monde passé, et sont fortement insérées dans le monde des « choses ».

« L'attachement au sol, l'attachement au pays (d'où leur nom de paysan), tel paraît

bien être le mobile ou le motif essentiel, qui explique qu'ils ne veuillent pas quitter le

\footnotetext{
${ }^{19}$ HaLBWACHS, 1964 (1938), 45.

${ }^{20}$ Ibid.

${ }^{21}$ GHISLAIN, STEINER, 1995.
} 
petit coin où ils sont nés, où ils ont pris racine, où leur famille vit depuis un temps qui leur paraît indéfini ${ }^{22}$.

Ils sont viscéralement attachés à la terre à laquelle ils lient leur identité de groupe, lequel est resté le gardien du monde passé et ne s'est pas confondu avec le reste de la population. D'où une vie collective qui s'appuie sur cette double conscience d'être quelque peu à l'écart, mais de représenter un foyer de tradition. Occuper un sol qui, en quelque sorte, fait partie d'eux-mêmes et du groupe lui-même les conforte dans le sentiment d'une certaine immuabilité de l'existence, par delà la succession des générations. Ainsi,

« La vie du groupe paysan est profondément engagée, et tout entière, dans la nature.

De là une saveur et une âpreté particulières, plus de spontanéité, d'élan primitif et même de sauvagerie (...). C'est une vie collective à la fois très forte et très simple, ou très simplifiée » $(79)$.

Corrélativement, cette vie plus simple et plus primitive limite à son strict minimum la vie sociale, c'est-à-dire la capacité à se regrouper, à laisser une place à la vie collective, à la laisser s'insinuer dans les désirs et les aspirations communes. Car le paysan a plutôt la volonté de ne pas quitter son village et sa terre, une tentation de repli sur soi et la faculté de rester à travailler la terre pour un revenu faible, dans des conditions parfois terribles. Les paysans vivent isolés, soucieux du bien-être de leur seule famille, assène Halbwachs :

«Âpreté et égoïsme, individualisme à la base comme au sommet. On est économe, frugal (...). On ne se soucie pas des autres. Il n'y a pas une tendance naturelle à s'associer, même entre les habitants d'un même village, d'une même région, dans une pensée d'intérêt commun » (81).

De la sorte c'est une forme plus instinctive d'existence qui les caractérise, parce que portant moins la marque de l'esprit collectif mais pour autant qu'il éloigne des instincts les plus « naturels », ou les plus « sauvages », animaux.

Il faut voir dans l'explication de cette existence instinctuelle le résultat de l'extrême isolement des maisons, l'absence d'ordre et d'organisation dans la façon dont elles se distribuent, en vertu du fait qu'elles sont conçues comme le centre de leur exploitation familiale. Le village n'est donc pas envisagé comme une unité, ce qui a pour effet de lui conserver

«Un aspect inorganique et désordonné ${ }^{23}$, où s'exprime, sinon l'isolement et

l'indépendance des paysans, du moins le relâchement du lien social dans les groupes ruraux $\gg{ }^{24}$.

Ce désordre est dû à l'influence de la mentalité paysanne, qui ne pense pas sa maison indépendamment de ses autres biens.

«Aussi la conscience collective, en de tels groupes, est-elle beaucoup plus diffuse $»(42)$.

Il faut donc sans doute comprendre que là où la civilisation fait son œuvre, dans la marche vers la société à « solidarité organique » de Durkheim, pourvu qu'à côté de la

\footnotetext{
${ }^{22}$ HALBWACHS, 1964 (1938), 65.

${ }^{23}$ C'est nous qui soulignons.

${ }^{24}$ HalBWACHS, 1970b (1912), 43.
} 
tendance à l'individualisation l'esprit collectif continue d'imprimer sa marque, il permet que se crée une conscience de groupe plus puissante parce que moins entravée par la vie instinctuelle, condition de cette « vie psychique plus haute » dont parlait déjà Durkheim ${ }^{25}$. C'est pourquoi les paysans sont en quelque sorte les exclus de la marche vers une autre civilisation. En cela, ils sont comparables aux ouvriers, à ceci prêt que ces derniers, plus engagés dans le monde social, font plus figure de « vaincus » de la lutte pour la vie que les paysans, qui en sont comme d'emblée écartés en tant que « survivance » de la civilisation passée.

\title{
Les ouvriers et l'asservissement à la matière
}

\begin{abstract}
« Rassemblés dans les mines, réunis en équipes autour des machines, les ouvriers se présentent comme des groupes soumis à une discipline. Ils exécutent mais ne commandent pas $»{ }^{26}$.
\end{abstract}

En somme, ils ne contrôlent pas « la marche et le rythme » de leur travail (135), tandis que dans nos sociétés occidentales « la loi du travail et de l'activité régulière, ininterrompue, intense parfois et qui oblige l'homme à aller jusqu'au bout de ses forces, s'est de plus en plus imposée (146). Dans les locaux industriels, «l'ouvrier perd en partie sa personnalité » (175). Si bien que de manière générale, la maind'œuvre ouvrière s'adapte plus aux tâches disponibles qu'elle ne les choisit.

\section{Il en résulte que l'hérédité des parents joue un rôle important, et souvent ceux qui exercent un métier depuis longtemps répugnent à en changer. \\ « Il n'est guère douteux, en effet, que toutes les activités ouvrières soient à la base d'instincts ou de dispositions humaines. Acuité sensorielle, sûreté des mouvements, résistance nerveuse, force musculaire, ingéniosité, agilité, c'est là tout un ensemble de facultés actives et sensibles qui nous semblent développer simplement des tendances faisant partie de la nature humaine, et qui étaient en elle avant de se manifester dans un métier, à l'état embryonnaire » (142).}

- Les traits distinctifs de la psychologie ouvrière sont donc aussi à chercher du côté des types sociaux les moins délicats et les moins développés :

«Une disposition d'esprit de bonne heure plus grave, comme elle se développe dans l'isolement, une pensée à la fois plus lente et plus fruste, une sensibilité à la fois plus refoulée et plus émoussée ; plus d'insouciance apparente, peut-être, leur sérieux s'étant dépensé dans l'exercice d'un travail qui oblige leur pensée à sortir nettement du cercle social ; à la fois moins de sociabilité et plus de solidarité, c'est-à-dire un certain détachement vis-à-vis de la société et de ses coutumes ${ }^{27}$.

- En un mot, les ouvriers constituent un groupe que sa condition maintient en deçà d'une certaine « socialité », parce qu'asservi à la matière qu'ils travaillent :

«Le travail oblige, pendant la plus grande partie de la journée, de rester en contact avec la matière inerte, avec des matières malléables et fragiles (...) avec des matières dures et résistantes (...) avec des matières dangereuses, avec des matières malpropres (...). Il en résulte que le travailleur de l'industrie est isolé du monde, la plupart du temps, et à la différence des autres agents de la vie économique,

\footnotetext{
${ }^{25}$ DURKHEIM, 1898, 48.

${ }^{26}$ HALBWACHS, 1964 (1938), 132.

${ }^{27}$ HALBWACHS, 1970b (1912), 76.
} 
commerçants, employés, contremaîtres, caissiers, etc., que leur travail met en rapport avec des personnes et n'oblige pas à sortir des groupes humains $»{ }^{28}$.

Quand ils sont réunis en groupes dans le cadre de leur travail, les ouvriers associent "leurs forces et leurs mouvements physiques», cette coopération est technique, si bien que les rapports qui s'établissent entre eux sont « machinaux », et « n'intéressent ni la pensée, ni le sentiment ${ }^{29}$.

Il n'est sans doute pas exagéré d'avancer que dans cette optique, privés des ressources cognitives, psychologiques et sociales qui feraient d'eux une organisation sociale pleinement développée, les ouvriers ne peuvent atteindre un degré de conscience de soi et des autres comparable à celui des bourgeois.

« Seul dans la société (avec le paysan), l'ouvrier exerce son travail directement sur la matière inanimée et se trouve, par suite, en contact quotidien avec elle. Tous les autres membres de la société sont mis, par le fait de leur travail, en relations avec des hommes ou avec une matière humaine, qu'ils exercent des professions libérales, qu'ils soient commerçants ou même employés. Les uns donnent l'impulsion, les ordres, les conseils et les autres les rédigent, les transmettent, en assurent l'exécution ou en surveillent les résultats. Seuls les ouvriers se bornent à recevoir des ordres ou des instructions, et, lorsqu'ils les exécutent, n'agissent que sur la matière, non plus sur les hommes $\gg{ }^{30}$.

Tout comme la société élimine impitoyablement ses canards boiteux, en les poussant au suicide, afin de s'adapter aux conditions d'une vie plus compliquée où la lutte pour la vie est plus intense, elle «reporte hors d'elle» «toute une classe d'hommes délégués au travail matériel, a su fabriquer des outils à manier des outils $\gg{ }^{31}$.

- Cette « entrave » qu'elle impose aux ouvriers dans le passage de la nature à la culture, pourrait-on dire, explique paradoxalement l'attachement très fort de ceuxci à leur métier, la volonté de consacrer la majeure partie de son temps à l'effort, et le désarroi qui s'empare d'eux dès lors que se pose la question de savoir comment utiliser ses loisirs

«C'est que la vie est organisée en vue du travail et non du reste. Les hommes ont perdu, n'ont jamais pu développer, d'autres goûts et d'autres besoins ${ }^{32}$.

Cela commande aussi des aspirations - dont les dépenses mesurées par les budgets - familles sont un indicateur comme on sait - qui se résument à la volonté d'améliorer si possible sa condition, ou au moins de conserver le niveau de vie acquis.

\section{Une théorie de l'action économique ouvrière}

Ce point de vue, en germe chez Halbwachs dès le travail sur La classe ouvrière et les niveaux de vie (1912), recoupe en grande partie la théorie de l'action de Simiand,

\footnotetext{
${ }^{28}$ HalBwaCHS, 1964 (1938), 142.

${ }^{29}$ Ibid.

${ }^{30}$ HalBWACHS, 1970b (1912), 75.

${ }^{31}$ Ibid.

${ }^{32}$ Halbwachs, 1964 (1938), 147.
} 
et dont on trouve la version aboutie dans sa recherche sur Le salaire (1932). Cette théorie a déjà été sériée et commentée ${ }^{33}$, aussi ne le reprenons-nous pas en détail.

Ce qui nous intéresse ici, c'est que la classe des salariés, par son opposition à une compression des salaires nominaux, crée une inertie qui incite les entrepreneurs à l'innovation. Autrement dit, si pour Simiand ouvriers et patrons manifestent des tendances communes, et si les uns et les autres sont obligés de composer avec la force relative de ces différentes tendances, dans cette interaction conflictuelle les ouvriers subissent plus qu'ils n'impulsent. On trouve du reste de belles pages d'Halbwachs dans son Esquisse, qui reprend une à une les tendances mises en évidence par Simiand, et les illustre dans ce sens ${ }^{34}$. Ainsi, si les ouvriers ont une «tendance à conserver le même gain » qui passe par une farouche défense du montant de leur salaire nominal, c'est parce que ce salaire incarne non seulement la valeur de leur travail, mais celle «de la personne humaine tout entière engagée dans la production ${ }^{35}$. S'ils ont tendance à ne pas augmenter leur effort c'est qu'ils ont l'impression que primes et méthodes d'organisation du travail destinées à améliorer le rendement sont fixées sans qu'on se rende compte du sacrifice qu'est pour eux ce travail supplémentaire, etc. ${ }^{36}$.

\section{IV - Au sommet de la hiérarchie sociale : des groupes au type social « évolué »?}

\section{Bourgeois et concurrence}

À cette place figurent ceux qu'Halbwachs identifie comme les bourgeois et les capitaines d'industrie. Parmi les représentations collectives et les aspirations qui les caractérisent, on trouve le sentiment d'un devoir de responsabilité.

« Membres d'une classe élevée, il leur apparaît que «bourgeoisie » (de même que noblesse) oblige. Et ils veulent apporter devant les autres la preuve qu'ils sont dignes de faire partie de cette classe. Souci de respectabilité bourgeoise, qui leur commande d'agir suivant les règles de la morale courante dans leur milieu, où il est admis que l'homme doit faire tout ce qu'il peut pour accroître sa richesse, mais en se conformant aux usages et traditions sur lesquelles repose le prestige de la classe elle-même » (118-119).

Ainsi, sur un vieux fond de morale traditionnelle, la bourgeoisie a développé des « qualités » qui l'ont rendue capable de s'adapter aux conditions nouvelles de la vie sociale :

«Ce n'est pas l'argent tout seul, les réserves d'argent dont ils pouvaient disposer, qui leur eussent donné ces qualités. Ces qualités, plutôt, les rendirent capables de trouver les fonds nécessaires pour les mettre en œuvre (110).

- Ce que la bourgeoisie caractéristique de la société capitaliste moderne exprime le mieux, c'est un ensemble de représentations collectives issues du nouveau régime

\footnotetext{
${ }^{33}$ Ghislain, Steiner, 1995 ; Marcel, 2001.

${ }^{34} \mathrm{Ph}$. Steiner signale un travail similaire d'illustration des tendances de l'action dégagées par Simiand, par des calculs de budgets de familles, dans la thèse de 1912 (STEINER, 2005, 135).

${ }^{35}$ HALBWACHS, 1964 (1938), 137-138.

${ }^{36}$ Ibid., 139 et suiv.
} 
économique auxquelles ces agents estiment qu'ils doivent obéir. Parmi les motivations qui émergent de ces représentations on trouve bien sûr la préoccupation du gain et de son accroissement indéfini : « le devoir de s'enrichir, non pour jouir de plus de biens que l'on consommera, mais pour avoir plus de moyens de s'enrichir encore : tel est le nouveau devoir, qui, comme un impératif catégorique, est à la base de la morale capitaliste » (97). Ce motif, précise Halbwachs, citant Schumpeter et Weber, se décline en une tension perpétuelle, un déploiement continu de volonté et d'énergie.

Au demeurant, ce qui caractérise le mieux la mentalité de cette classe sociale, c'est l'esprit de lutte et de concurrence, notions aux côtés de laquelle on trouve l'individualisme et le libéralisme. La concurrence est devenue la loi de la production et de la vente. Il en résulte des dispositions "réalistes» et "égoïstes » qui sont comme « une réaction nécessaire et surtout comme une arme dans la lutte pour la vie et surtout pour les gains » (103).

- La bourgeoisie se définit enfin par la rareté de ses qualités ${ }^{37}$, car elle a la fonction éminemment sociale qui consiste à prendre les grandes décisions qui vont guider l'activité des hommes, bref en un mot réaliser l'adaptation de la vie sociale aux fins de la société. Commandant aux hommes, impulsant les grandes évolutions de la technique, dont le rôle est de mettre un peu d'ordre dans un monde social mouvant, grâce à un faisceau de règles générales et fixes, le bourgeois « a une connaissance sociale des goûts, des manières d'être, dans les différentes parties du monde et de la société » (49). L'esprit d'entreprise, qui suppose l'esprit d'extension, de renouvellement, de transformation, participe de cette fonction d'adaptation qui suppose aussi la conscience d'une responsabilité collective.

En un mot la classe bourgeoise se caractérise par des représentations et des aspirations qui la placent au cour de la vie sociale, là où l'on est le moins dépendant des contraintes du monde matériel, et plus engagé dans la vie sociale.

\section{« Mettre en œuvre et monnayer l'avenir »}

Dans le travail de Simiand c'est la place de «premier moteur » de la vie collective que les classes bourgeoises tiennent dans l'évolution économique général. Celle-ci prend la forme de développement en cycles alternés, processus par lequel la société se développe et s'adapte à ses conditions d'existence. Ceci découle de ce que dans les sociétés modernes ce sont les faits économiques qui prédominent, comme on l'a vu.

Or, ce sont les lanceurs d'affaires, capitaines d'industries et autres magnats qui conditionnent cette alternance de phases. Assumant des fonctions de direction, prenant le risque de lancer de nouvelles productions, de réaliser aussi des opérations commerciales, industrielles, financières, bref mettant en œuvre des moyens de façon économique, ils assument une fonction éminemment sociale reliée à des fins collectives : " réaliser l'ensemble d'action économique correspondant le mieux à la fin assignée $"{ }^{38}$. En dernier recours, il s'agit de dégager un profit pour développer un peu plus l'entreprise. Cette fonction est empreinte d'une lourde

\footnotetext{
${ }^{37}$ HALBWACHS, 1939, 51 .

${ }^{38}$ SIMIAND, 1930, 417.
} 
responsabilité car elle a une généralité croissante dans une société d'échange complexe.

La mise en œuvre réalisée par la bourgeoisie permet que soit possible l'échange, au cours duquel s'établissent des prix, qui sont des appréciations. En rendant possible la création de la richesse et son attribution au cours de l'échange, la bourgeoisie fait en sorte que «la valeur économique des choses » ait " une certaine correspondance reconnue avec un besoin humain » (428). On peut donc dire que la classe supérieure est chez Simiand détentrice d'un savoir socialement constitué, qui se déploie au travers de certaines actions économiques essentielles au fonctionnement de la société. L'entrepreneur fait des « prévisions », « des raisonnements sur des éléments multiples qui ne peuvent être connus » et émet « des jugements dont on ne peut donner de règle simple matérielle et automatique» (58). Bref, il fait des prévisions non probabilisables en tâchant de se faire une idée des possibilités ultérieures du marché. Or, comme on sait, ces aptitudes relèvent plus d'un « instinct social », et débordent le domaine de la conscience claire. On peut donc logiquement penser qu'elles se développent au mieux dans le milieu social "porteur» que constitue la classe bourgeoise, placée au cœur de ce qui fait la vie collective dans les sociétés d'échange complexe ${ }^{39}$.

Parmi les actions qui en découlent, on trouve la fonction économique essentielle, qui consiste à aider à faire passer le système économique d'un stade présent à un stade futur, en réalisant par l'emprunt et le crédit un afflux de moyens monétaires. C'est, explique Simiand,

«Un processus qui consiste à réaliser d'avance des moyens tirés de ce futur (...)

Autrement dit, la société humaine en ces phases, sans l'avoir raisonné ni même toujours compris, découvre et pratique ce moyen essentiel et effectif d'enrichissement qui est, en propres termes, de monnayer l'avenir $\gg{ }^{40}$.

Emprunter permet de donner une impulsion à la réalisation anticipée de la richesse à venir, et constitue dès lors le moyen privilégié qu'ont les hommes de développer leur action et d'inscrire les résultats de ce développement dans l'avenir, en accroissant biens et richesses. Prix et salaires sont autant de représentations collectives qui découlent d'appréciations collectivement constitués, signaux éminemment complexes qu'il faut tenter de déchiffrer et qui impliquent que se constituent « une croyance et une foi sociales » dont la monnaie est le vecteur ${ }^{41}$ et que la classe bourgeoise crée.

En d'autres termes, celle-ci est la détentrice privilégiée d'un «savoir» qui lui permet d'exprimer au mieux l'instinct social qui anime la société : anticiper sur l'avenir ${ }^{42}$; ou si l'on préfère, elle incarne de façon privilégiée la « raison collective » qui commande le progrès économique, lequel est réductible à une forme évoluée de

\footnotetext{
${ }^{39}$ Cela explique aussi sans doute pourquoi il n’y a pas à proprement parler chez Simiand de théorie de la classe moyenne. La $30^{\mathrm{e}}$ leçon de la $2^{\mathrm{e}}$ année du Cours d'Économie Politique, consacrée à la question fait plus état d'une énumération que d'une véritable définition, comme le remarque judicieusement HALBWACHS (1939, 33). La place des classes moyennes dans le mouvement du cycle économique n'est pas clairement identifiable, de fait.

${ }^{40}$ SIMIAND, 1932b, vol. 2, 519.

${ }^{41}$ MARCEL, STEINER, 2006, 214.

${ }^{42}$ Quoique dans une autre perspective, $\mathrm{Ph}$. Steiner montre que cette question de «l'attente » est au cœur du fonctionnement de la vie sociale, dans la sociologie religieuse de Mauss aussi bien que dans la sociologie économique de Simiand. S'efforçant d'expliquer cette convergence de points de vue, il y voit, de façon subtile, deux branches dérivées d'un programme de recherche élaboré par Durkheim (STEINER, 2005).
} 
lutte pour la vie. On pourrait s'autoriser à y voir une illustration de ce « dénouement adouci » de lutte pour la vie, caractéristique des sociétés aux types sociaux évolués, qu'anticipait déjà Durkheim :

« La division du travail est donc un résultat de la lutte pour la vie : mais elle en est un dénouement adouci. Grâce à elle, en effet, les rivaux ne sont pas obligés de s'éliminer mutuellement, mais peuvent coexister les uns à côté des autres ${ }^{43}$.

Pendant la phase descendante du cycle, certains capitaines d'industrie, assoupis dans la recherche du luxe ostentatoire et improductif disparaissent, alors que les éléments neufs et entreprenants y sont incorporés, soit par associations économiques, soit au moyen d'alliances matrimoniales. C'est donc par le biais de ces «poussées instinctives » que, sur le mode de la création, la vie sociale se perpétue par une série d'adaptations successives dont les bourgeois sont les principaux agents ${ }^{44}$.

\section{Conclusion}

Au final, avec la lecture « organiciste » que nous proposons ici de la théorie des classes sociales selon Simiand et Halbwachs, il nous semble qu'on peut trouver quelques éléments pour expliquer entre autres que l'un et l'autre aient à ce point négligé les apports du marxisme, ou tout au moins la place du conflit révolutionnaire, dans leurs analyses. Si une relative conscience d'être " perdants », ou pour reprendre un terme plus moderne, un relatif sentiment d'aliénation peut caractériser la classe ouvrière dans leurs recherches, il reste que la place des uns et des autres est fondée en raison par la prise que la société exerce sur les différents groupes qui la composent. À ce titre, il n'y pas lieu de chercher à modifier l'ordre des choses par l'action, somme toute, artificielle de ce point de vue, d'un quelconque groupe révolutionnaire. Simiand et Halbwachs ne méconnaissent pas, bien sûr, le rôle et l'action des syndicats ouvriers. Ils consacrent du reste l'un et l'autre de longs développements à ces institutions. Mais dans cette optique, le syndicat n'est étudié et n'a de sens que dans la mesure où il contribue à œuvrer à l'amélioration ou au maintien de la condition de la classe ouvrière en garantissant le lien entre le travail et sa rémunération. Simiand est sur ce point très explicite :

« Mais là même où ces aspirations existent bien en fait et ont une importance, qui n'est pas niable ce qui importe du point de vue d'étude positive et d'abord, c'est de regarder à ce que font les hommes dans ces institutions plutôt qu'à ce qu'ils disent ou peuvent faire ou aspirent à faire. Or, en fait jusqu'à présent tous les pays, les associations syndicales ouvrières n'ont pas assumé d'autre rôle de façon positive que de maintenir ou améliorer la condition ouvrière comme telle $\gg{ }^{45}$.

La hiérarchie sociale se pense donc comme étant dans l'ordre des choses, le résultat d'une sorte de mouvement de l'histoire par lequel le social exprime sa perfection en organisant et en infléchissant la lutte pour la vie.

Sa conception valut notamment à Simiand de sévères critiques de la part de ses amis socialistes, heurtés par le caractère fataliste, et logiquement noninterventionniste de la théorie du cycle économique. En 1933 par exemple, le

\footnotetext{
${ }^{43}$ DURKHEIM, 1991 (1893), 253.

${ }^{44}$ Frobert, 1996, 161-62.

${ }^{45}$ SimiAND, 1930, 492.
} 
socialiste Georges Boris dénonce «l'irresponsabilité doctrinale » de la théorie du progrès par alternance d'ondes longues, « pulsations essentielles » qui constituent « la manifestation d'une loi biologique permanente qui régit notre société $»{ }^{46}$. Peut-être faut-il voir ainsi dans la présence persistante de cette analogie organiciste chez les durkheimiens une des explications du hiatus entre leurs engagements citoyens et leurs convictions de théoriciens.

Jean-Christophe MARCEL

Université de Paris IV-Sorbonne, France jean-christophe.marcel@paris-sorbonne.fr

\section{Bibliographie}

BlancKAerT C., 2004, La nature de la société. Organicisme et sciences sociales au XIX siècle, Paris, l'Harmattan.

Durkheim É., 1991 (1893), De la division du travail social, Paris, Presses Universitaires de France.

DURKHEIM É., 1898, Représentations individuelles et représentations collectives, Revue de Métaphysique et de Morale, 6, 273-302.

Frobert L., 1996, Une double interprétation des mouvements économiques, in GiLlard L., Rosier M., (dir.), François Simiand (1873-1935). Sociologie-Histoire-Économie, Paris, Éditions des Archives Contemporaines, 23-57.

Frobert L., 2000, Le travail de François Simiand, Paris, Économica.

Ghislain J.J., Steiner Ph., 1995, La sociologie économique, Paris, Presses Universitaires de France.

Guillo D., 2006, La place de la biologie dans les premiers textes de Durkheim : un paradigme oublié ?, Revue Française de Sociologie, 47, 3, 507-535.

HalbWachs M., 1930, Les causes du suicide, Paris, Alcan.

Halbwachs M., 1939, Les caractéristiques des classes moyennes, in ArON R., et al., Inventaires. Tome III : Les classes moyennes, Paris, Alcan, 28-52.

Halbwachs M., 1964 (1938), Esquisse d'une psychologie des classes sociales, Paris, M. Rivière.

Halbwachs M., 1970a (1938), Morphologie sociale, Paris, A. Colin.

HalbWachs M., 1970b (1912), La classe ouvrière et les niveaux de vie, Paris-Londres-New York, Gordon and Breach.

MARCEL J.Ch., 2001, Le durkheimisme dans l'entre-deux guerres, Paris, Presses Universitaires de France.

MARCEL J.Ch., 2004, Mauss et Halbwachs : vers la fondation d'une psychologie collective, Sociologie et Sociétés, XXXVI, 2, 73-90.

Marcel J.Ch., Steiner Ph., 2006, Présentation générale, in Simiand F., Critique sociologique de l'économie, Paris, Presses Universitaires de France, 1-25.

Simiand F., 1930, Cours d'économie politique. Tome 2 : années 1928-1929, Paris, Domat Montchrestien.

Simiand F., 1932a, Cours d'économie politique. Tome 1 : années 1930-1931, Paris, Domat Montchrestien.

\footnotetext{
${ }^{46}$ BORIS, in FrOBERT, 2000, 181.
} 
Revue d'Histoire des Sciences Humaines

Simiand F., 1932b, Le salaire, l'évolution sociale et la monnaie, Paris, Alcan (2 volumes).

SteINER Ph., 2005, L'école durkheimienne et l'économie, Genève-Paris, Droz. 